\title{
INFLUÊNCIA DA RESOLUÇÃO DO MDT EM MODELAGEM DE INSTABILIDADE DE VERTENTES: ANÁLISE PRELIMINAR
}

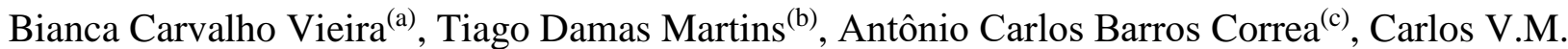 \\ Bateira $^{(d)}$ \\ (a) Departamento de Geografia/Universidade de São Paulo, biancacv@usp.br \\ (b) Departamento de Ciências Geográficas (BFP/FACEPE)/Universidade Federal do Pernambuco, \\ martins.td@gmail.com \\ (c) Departamento de Ciências Geográficas/Universidade Federal do Pernambuco, dbiase @ hotmail.com \\ (d) Riskam-CEG-UL/FLUP-UP (Portugal), carlosbateira@ gmail.com
}

\section{Eixo: GEOTECNOLOGIAS E MODELAGEM ESPACIAL EM GEOGRAFIA FÍSICA}

\begin{abstract}
Resumo
Um dos métodos utilizados para a indicação de áreas suscetíveis a escorregamentos rasos são os modelos matemáticos em bases físicas, que se apoiam, destacadamente, na topografia. Portanto, a geração de um modelo digital de representação do relevo é essencial, pois a partir deste é possível extrair as informações como área de contribuição e ângulo da encosta, consideradas fundamentais na sua aplicação. Este trabalho teve como objetivo realizar uma análise preliminar na resposta de um modelo em base-física quando alterado a dimensão (resolução) da célula que compõe o Modelo Digital de Terreno, elaborado a partir de dados topográficos convencionais (curvas-de-nível). Os resultados apontam que não houve significativa melhora na definição de áreas instáveis, quando das alterações da resolução do MDT.
\end{abstract}

Palavras chave: Escorregamentos Rasos; SHALSTAB; Serra do Mar.

\section{Introdução}

Movimentos de Massa são processos comuns na paisagem, em especial naquelas com relevo montanhoso e com registro de altos valores de precipitação. A ocorrência de tais processos tem trazido um constante desafio às pesquisas, pois podem acarretar em perdas sociais, econômicas e ambientais. De acordo com o banco de dados elaborado por Macedo e Martins (2015), são 773 eventos catalogados em 243 municípios, em 18 estados, constituindo um total de 3396 fatalidades entre 1988 e abril de 2015.

Destarte, frente aos desafios impostos, distintos métodos são desenvolvidos no intuito de indicar áreas potencialmente instáveis, sendo que um destes são os modelos matemáticos em bases físicas que descrevem fisicamente os processos por meio de equações matemáticas, desconsiderando a opinião subjetiva e direta do pesquisador. Tais modelos indicam a suscetibilidade sob diferentes cenários de uso e eventos de precipitação pluvial, por exemplo, independente da ocorrência anterior dos processos 
(CHORLEY, 1967; GUZZETTI et al., 1999). Dentre os modelos, o SHALSTAB (Shallow Landsliding Stability Model), desenvolvido nos Estados Unidos, a partir dos anos de 1990, tendo sua proposição e aplicação feitas por Montgomery e Dietrich (1994) e Dietrich e Montgomery (1998), calcula para cada unidade (célula) do terreno, seu potencial de instabilidade baseando-se em parâmetros físicos, como as propriedades físicas do solo, fluxo d'água em subsuperfície e, destacadamente a topografia, tendo como recorte espacial uma bacia hidrográfica. A proposição matemática deste modelo resulta da combinação da equação do talude infinito com um modelo hidrológico (MONTGOMERY e DIETRICH, 1994).

Assim em função da operação do SHALSTAB se apoiar sobretudo na topografia, é essencial a geração de um Modelo Digital de Terreno para extrair as informações como de área de contribuição e ângulo da encosta. Diversos trabalhos aplicando este modelo no Brasil, fizeram uso de MDT's com variados valores de resolução de células que compõem o grid (GOMES et al, 2005, ZAIDAN e FERNANDES, 2009; RAMOS e VIEIRA, 2015; dentre outros).

Gomes et al. (2005), por exemplo, buscaram comparar os resultados do modelo, a partir de dados topográficos em distintas escalas, 1:10.000 e 1:50.000. Os autores apontaram resultados eficientes, destacando que $70 \%$ das cicatrizes coincidiram com a classe Incondicionalmente Instável na escala 1:10.000, mas com decréscimo de acerto para escala 1:50.000, em que apenas 12\% das cicatrizes para esta mesma classe.

Em outras palavras, a resolução espacial do MDT é fundamental para o entendimento da espacialização das áreas potencialmente instáveis. Este é um tema discutido com recorrência na literatura, a exemplo de Zhang e Montgomery (1994) que avaliaram a reposta de MDT's com grids de distintas dimensões, variando entre 2, 4, 10, 30 e 90 metros, gerados a partir de pontos de elevação. Em seus resultados, os autores identificaram uma influência da dimensão das células nas respostas de modelos de base física para análise de fluxo, havendo uma melhora no grid de 10 metros em relação aos de 30 e 90 metros, mas nenhuma melhora significativa quando comparado com grids de 2 e 4 metros.

Com base no que foi tratado, este trabalho teve como objetivo analisar a resposta de um modelo em basefísica (SHALSTAB) em função da dimensão (resolução) da célula que compõe o Modelo Digital de Terreno. 
XVII Simpósio Brasileiro

de Geografia Fisica Aplicada

I Congresso Nacional

de Geografia Física
OS DESAFIOS DA GEOGRAFIA FÍSICA NA FRONTEIRA DO CONHECIMENTO

Instituto de Geociências - Unicamp

Campinas - SP

28 de Junho à 02 de Julho de 2017

\section{Materiais e Métodos}

A definição da área de estudo se deu diante da disponibilidade de dados cartográficos em distintas escalas, assim fora selecionada a Bacia Hidrográfica do Bom Brinquedo (BHBB), com $164.200 \mathrm{~m}^{2}$, localizada no Morro do Bom Brinquedo, município de Antonina/PR (Figura 1).

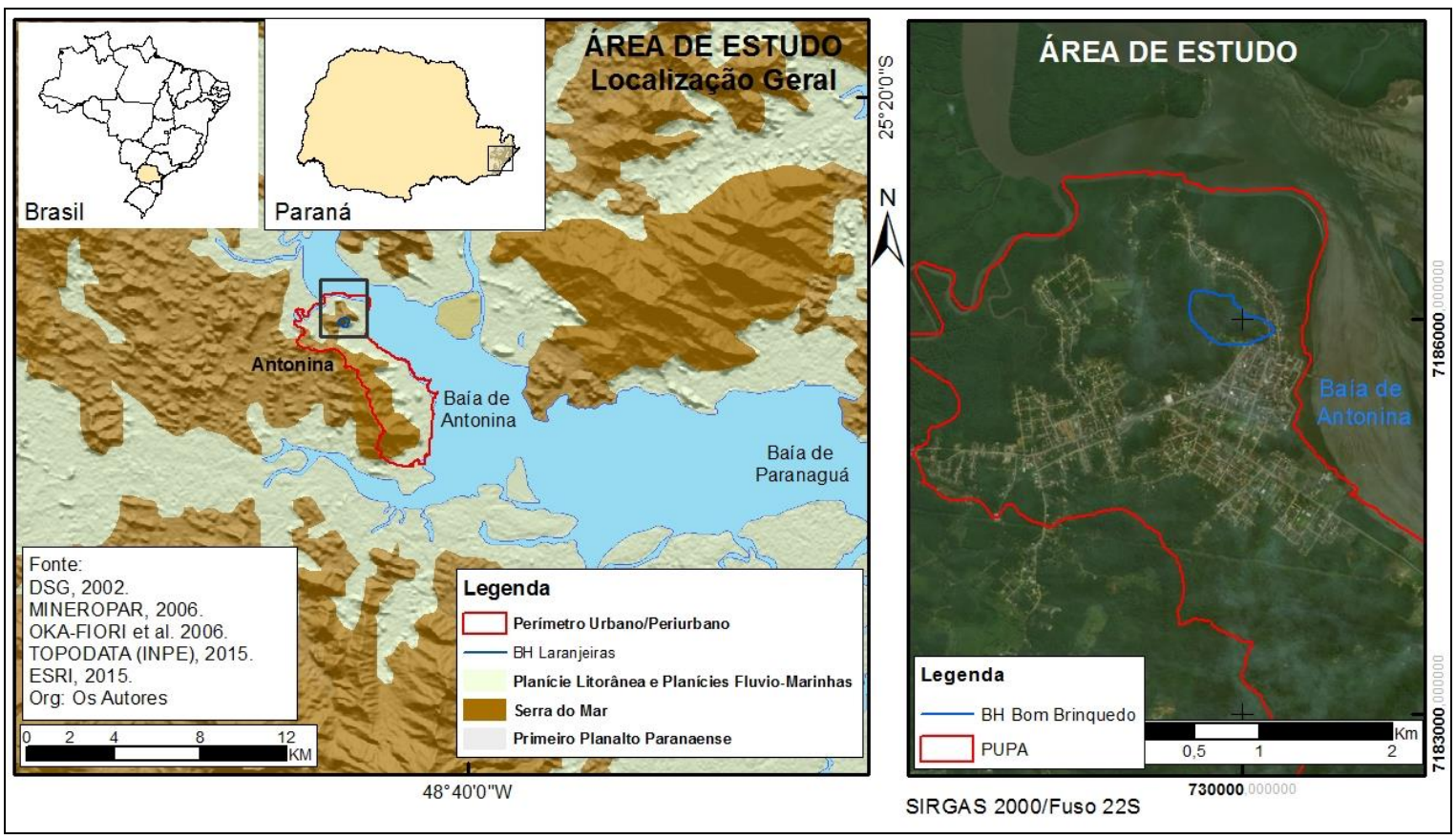

Figura 1: Localização da Área de Estudo.

Para a preparação das bases cartográficas foram utilizados dois conjuntos de dados que consistiram em: (a) curvas de nível com equidistância de $10 \mathrm{~m}$, provenientes de carta topográfica em escala 1:25.000 (DSG, 2002); (b) curvas de nível-mestras com equidistância de $5 \mathrm{~m}$, provenientes de dados topográficos em escala 1:2.000 do Plano Diretor Municipal (PMA, 2007).

De posse destes dados foram gerados dois Modelos Digitais de Terreno (MDTs), obtidos a partir das curvas de nível, utilizado a ferramenta Topo to raster, do módulo ArcMap (ArcGIS 10.3). A resolução do grid de cada MDT teve como critério de definição a equidistância entre as curvas, portanto os MDT's possuem resoluções de 10x10m e 5x5m.

A aplicação do modelo SHALSTAB, utilizando cada MDT, foi feita na sua versão disponibilizada como rotina empregada em Sistemas de Informação Geográfica- SIG (ArcView 3.3), de acordo com Dietrich e Montgomery (1998). O modelo SHALSTAB identifica áreas potenciais à ocorrência de escorregamentos rasos classificando-as entre Incondicionalmente Instável, passando por cinco classes intermediárias $(<-$ 
3.1, -3.1 a -2.8, -2.8 a -2.5, -2.5 a -2.2, > -2.2, dado em Log q/T) até a classe Incondicionalmente Estável. Seu resultado indica a razão da precipitação pluvial crítica necessária para a ruptura (q) pela transmissividade do solo (T) (MONTGOMERY e DIETRICH, 1994; DIETRICH e MONTGOMERY, 1998).

Foram utilizados os mesmos valores dos parâmetros físicos do solo nos dois MDTs, a saber: Ângulo de Atrito: $31^{\circ}$; Peso Específico $(P s): 2600 \mathrm{~g} / \mathrm{cm}^{3}$; Profundidade $(Z): 1 \mathrm{~m}$; e Coesão $\left(c^{\prime}\right)$ : zero. Estes valores foram obtidos por meio de testes realizados por Martins (2016) a partir de um conjunto de amostras in situ obtidas por Lopes (2013), em duas trincheiras junto a borda de uma cicatriz de escorregamento do Morro do Bom Brinquedo. Por fim, foram contabilizados o número de células de cada classe, o que permitiu verificar as suas frequências.

\section{Resultados e Discussões}

Os resultados mostraram que a distribuição da classe Incondicionalmente Instável foi similar em ambos os MDTs (Figura 2), marcadamente nos trechos médios das vertentes, havendo um claro aumento de células desta classe para o MDT de grid 5x5m, o que pode ser explicado pelo maior número de células que compõem a bacia.

Outrossim, o percentual de frequência das classes também repetiu a tendência similar em ambas as bacias, apresentando uma maior ocorrência de células nas classes Incondicionalmente Estável, Log q/T >-2,2, seguido pela classe Incondicionalmente Instável (Figura 3).

Em particular nota-se que a classe Incondicionalmente Instável registrou uma frequência de 12,48\% das células, utilizando o MDT de grid 10x10m, e um percentual ligeiramente maior, de 16,56\% utilizando o MDT de grid de 5x5m. As classes Log q/T >-2,2 e Incondicionalmente Estável registraram o maior número de células em ambos os MDTs: 42,08\% e 45,09\% (grid 10x10m) e 36,72\% e 46,11\% (grid $5 \times 5 \mathrm{~m})$.

Uma observação a ser destacada é a ínfima ocorrência de células nas classes intermediárias nos dois MDTs, havendo registro de valor zero, no caso do grid 10x10m e, de maneira geral, inferiores a $1 \%$ para ambos. Martins et al. (2016), verificou valores muito baixos nestas mesmas classes, para a mesma bacia hidrográfica ao realizar um conjunto de testes sobre a influência de MDTs, com resolução de 1x1m, originados de distintos dados cartográficos, verificou valores muito baixos nestas mesmas classes, para a mesma bacia hidrográfica. 


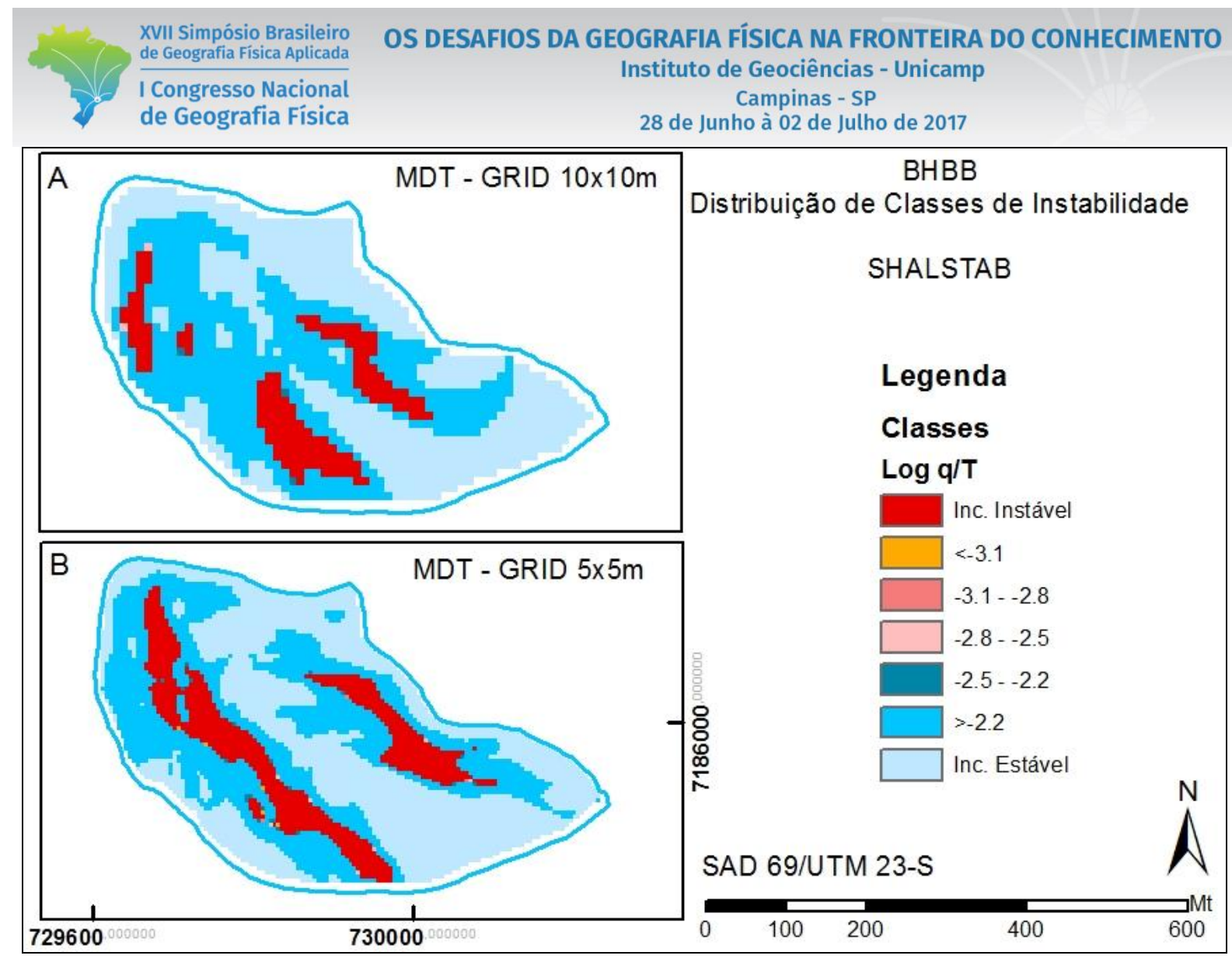

Figura 2: Mapa de classes de instabilidade da BHBB, dadas pelo SHALSTAB, considerando A) MDT-GRID 10x10m; e B) MDT-GRID 5x5m.
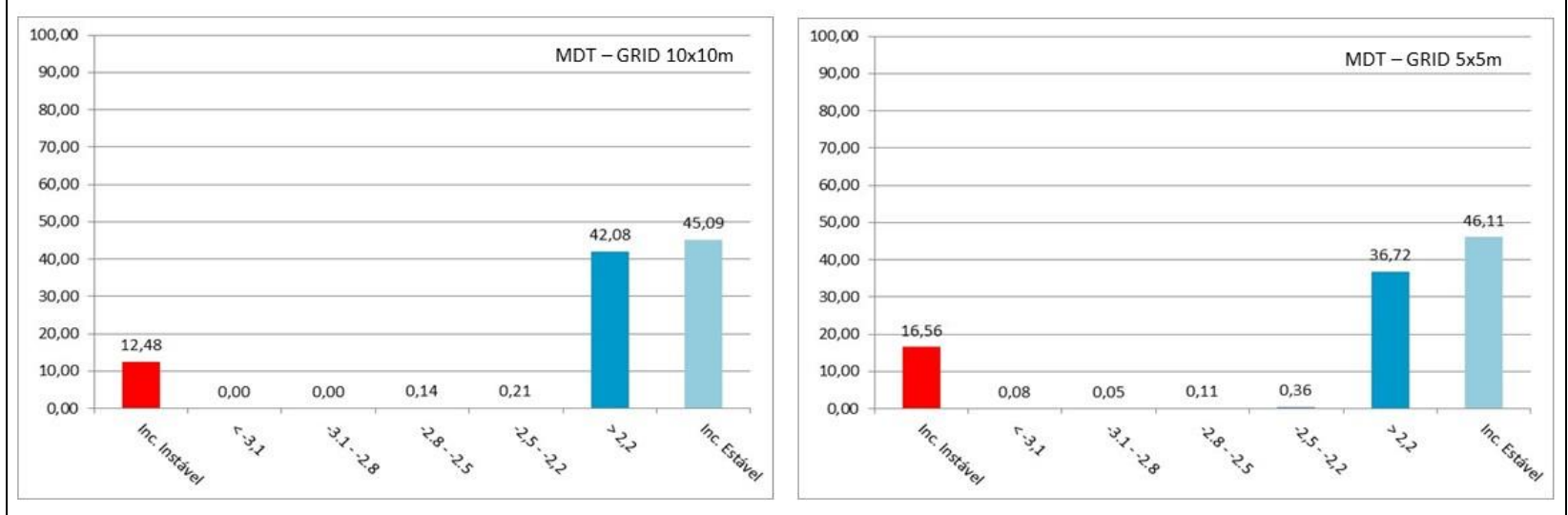

Figura 3: Gráficos de frequência de classes do modelo SHALSTAB para a BHBB, considerando o MDT-GRID 10x10m e MDT-GRID 5x5m.

Outra indagação diante dos valores obtidos, refere-se às classes que devem ser consideradas mais instáveis dependendo da dimensão da célula do grid. A exemplo do SHALSTAB, Dietrich et al. (2001) apresentaram uma discussão na qual questionam qual, ou quais, dentre as sete classes do modelo e, 


\section{OS DESAFIOS DA GEOGRAFIA FÍSICA NA FRONTEIRA DO CONHECIMENTO \\ Instituto de Geociências - Unicamp \\ Campinas - SP \\ 28 de Junho à 02 de Julho de 2017}

conforme a qualidade dos dados do MDT, devem ser consideradas classes de instabilidade. Os autores apontaram, para um MDT com grid de 5 metros, como sendo mais adequado considerar como instável apenas as duas primeiras classes do modelo (Incondicionalmente Instável e $\log \mathrm{q} / \mathrm{T}<-3,1$ ). Por conseguinte, as demais classes devem ser consideradas quanto maior a dimensão da célula do grid (Dietrich et al., 2001).

\section{Considerações Finais}

Este trabalho analisou, preliminarmente, a influência da resolução de dois Modelos Digitais de Terreno (10x10 e 5x5m) na identificação e na distribuição das vertentes instáveis, dadas pelo modelo matemático de base-física SHALSTAB.

Os resultados mostraram que não houve significativa melhora considerando os diferentes MDTs quando comparado a distribuição de células entre as sete classes do modelo, havendo ainda classes com valores inferiores a $1 \%$ em ambos os testes. Deste modo, aponta-se que a melhora da resolução, aumentou o número de células definidas como Incondicionalmente Instáveis, mas não significou o aprimoramento direto na resposta do modelo de análise de instabilidade.

Trabalhos futuros poderão aprimorar os testes verificando a coincidência destas classes com cicatrizes de escorregamentos previamente mapeadas, o que permitirá obter índices de acertos, a exemplo da correlação das áreas das cicatrizes com as classes instáveis do modelo.

\section{Agradecimentos}

A FACEPE (BFP) pelo auxílio financeiro e aos revisores anônimos do SBGFA/CNGF pelos comentários e sugestões.

\section{Bibliografia}

CHORLEY, R. J. Models in Geomorphology. In R. J. H. CHORLEY, P. (ed) Models in Geomorphology. Methuen \& Co., Londres, pp. 43-96. 1967.

DIETRICH, W. E.; MONTGOMERY, D. R., SHALSTAB: A Digital Terrain Model for Mapping Shallow Landslide Potential. In: National Council for Air and Stream Improvement, 1998. 
DIETRICH, W.E.; BELLUGI, D.; ASUA, R.R. Validation of Shallow Landslide Model, SHALSTAB, for Forest Management. In: Land Use and Watershed: Influence on Hydrology and Geomorphology in Urban and Forest Areas. Water Science and Application. American Geophysical Union: Vol. 2. P: 195-227. 2001.

DSG. Diretoria de Serviços Geográficos. Carta Topográfica Antonina. Folha SG.22-X-D-II-4-SO. 2002.

ESRI. ArcGis, Spatial Analyst, 3D Analyst. versão 10.3. Environmental Systems Research Institute, 2015.

GOMES, R. A. T.; GUIMARÃES, R. F.; CARVAlHO JÚNIOR, O. A.; FERNANDES, N. F. Análise de um Modelo de Previsão de Deslizamentos (Shalstab) em Diferentes Escalas Cartográficas. Solos e Rochas, São Paulo, v. 1, p. 85-97, 2005.

GUZZETTI, F.; CARRARA, A.; CARDINALI, M.; REICHENBACH, P. Landslides hazard evaluation: a review of current techniques and their application in a multi-scale study, Central Italy. Geomorphology, 31: 181-216. 1999.

LOPES, F.C.A. Avaliação da Influência da Distribuição Espacial da Espessura do Solo e do Nível Freático na Estabilidade de Encosta. 126p. Dissertação (Mestrado em Geografia) - Setor de Ciências da Terra, Universidade Federal do Paraná, 2013.

MACEDO, E.S. e MARTINS, P.P.D. Análise do banco de dados de mortes por deslizamentos do Instituto de Pesquisas Tecnológicas (IPT). In: Congresso Brasilerio de Gelogia De Engenharia e Ambiental, 15.,2015, Bento Gonçalves. Anais... São Paulo: ABGE, 2015. cd-rom. 7p.

MARTINS, T.D. Análise de Modelos Digitais de Terreno na Identificação de Áreas Suscetíveis a Escorregamentos Rasos 148p. Tese (Doutorado em Geografia) - Setor de Ciências da Terra, Universidade Federal do Paraná, 2016.

MARTINS, T.D.; OKA-FIORI, C; VIEIRA, B.C.; MONTGOMERY, D.R. Assessment of Lidar-derived DTMs for landslide susceptibility mapping: Application in the Brazilian subtropical forest. In Landslides and Engineered Slopes. Experience, Theory and Practice. 1ed.: CRC Press, p. 1389-1392. 2016.

MINEROPAR. Minerais do Paraná S.A. Carta Geomorfológica Curitiba -SG.22-X-D. Curitiba: MINEROPAR. 2006.

MONTGOMETY, D. R.; W. E. DIETRICH. A physically based model for the topographic control on shallow landsliding. In: Water Resources Research 30: 153-1171. 1994.

OKA-FIORI; SANTOS, L.J.C.; CANALI, N. E.; FIORI, A.P.; SILVEIRA, C.T.; SILVA, J.M.F.; ROSS, J.L.S. Atlas Geomorfológico do Estado do Paraná: escala base 1:250.000, modelos reduzidos 1:500.000. Minerais do Paraná MINEROPAR, Curitiba, 59 p. 2006.

PMA. Prefeitura Municipal de Antonina. Plano Diretor Municipal. 2007.

DOI - 10.20396/sbgfa.v1i2017.2193 - ISBN 978-85-85369-16-3 
TOPODATA. Banco de Dados Geomorfométricos do Brasil. Disponível em: http://www.dsr.inpe.br/topodata/. Acesso em: Janeiro de 2015.

VIEIRA, B.C.; RAMOS, H. Aplicação do Modelo SHALSTAB para Mapeamento da Suscetibilidade a Escorregamentos Rasos em Caraguatatuba, Serra do Mar (SP). Revista do Departamento de Geografia. V29, p. 161-174. 2015

ZAIDAN, R.T.; FERNANDES, N.F. Zoneamento de Suscetibilidade a Escorregamentos Rasos em Encostas Aplicado à Bacia de Drenagem Urbana do Córrego do Independência - Juiz de Fora (MG). In: Revista Brasileira de Geomorfologia. v.10, $\mathrm{n}^{\mathrm{o}}$ 2, p. 57-76, 2009.

ZHANG, W; MONTGOMERY, D.R. Digital elevation model grid size, landscape representation, and hydrologic simulations, In Water Resources Research, v30, Issue 4, pg. 1019-1028. 1994. 